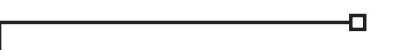

口-

The dynamic behavior in the clamped edge stress of structures is not yet fully understood clearly; also, clamped structures involve uncertainty. This research presents a numerical and analytical study of clamped edge stress behavior due to the load imposed by the chip-cutting tool on a workpiece. Clamping system, which is made of $\mathrm{H}-13$ steel and machining workpiece made of AISI 8620 steel are analyzed. The maximum clamped edge stress is analyzed through dynamic response, considering the machined part as a cantilever beam, involving the constitutive relations as well as the compatibility equations. The central differential equation of motion leads us to determine the modal stresses that are a primary characteristic of the structure and that are also distributed in it. Once the modal stress has been determined as well as the maximum amplitude at the free end of the specimen to be machined, it is possible to calculate the maximum clamped edge stress that is generated between both the specimen and the clamping system. Finally, a numerical analysis of the clamping jaw is performed for the discretised system and analyzed separately using the finite element method. Clamped edge stresses are assessed through a modal study using a set of numerical simulations to corroborate the modal stress estimated analytically. The results show that the clamped edge stress in the clamping system is a considerable influence in the design parameters of the structure. Therefore, complete knowledge of the dynamic response of the clamping system will lead to better structural design with the possibility of using different materials for the same purpose

Keywords: clamped edge stress, modal analysis, dynamic response, clamping system, modal stresses

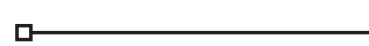

\section{CLAMPED EDGE STRESS ANALYSIS IN H-13 STEEL}

\author{
Carlos de Ia Cruz Alejo \\ PhD, Professor* \\ E-mail: cdelacruza@ipn.mx
}

Christopher R. Torres-San Miguel

PhD, Professor

Department of Mechanical Engineering

Postgraduate Studies and Research Section

National Polytechnic Institute

Lindavista ave., Mexico City, Mexico, 07738

E-mail: ctorress@ipn.mx

Juan C. Paredes-Rojas

$\mathrm{PhD}$, Professor

Mexican Center for the Cleanest Production

National Polytechnic Institute

Ticomán, Mexico City, Mexico, 07340

E-mail: paredesrojasjc@gmail.com

Ferna ndo E. Ortiz-Hernánde z

$\mathrm{MsC}$ *

E-mail: fernandoelih@gmail.com

*Department of Mechanical Engineering

Higher School of Mechanical and Electrical Engineering

National Polytechnic Institute

Santa Ana ave., Mexico City, Mexico, 1000

\section{Introduction}

In mechanical engineering, analysis of mechanical vibrations is significant for the design of mechanical elements. Structural vibration behavior is the leading cause of failure in the elements because of dynamic loads that induce vibrations in structural elements [1]. The dynamic behavior of a structure in a machine is typically analyzed as a cantilever beam since it is the simplest model of a structure. Beam models have solved many engineering problems in the past two centuries. The study of the vibration of beams subjected to loads is a classic topic discussed in many technical publications $[1,2]$. The natural frequencies of axially loaded beams, widely used in many macro and microstructures, are of practical interest in many applications. For example, for structural health monitoring of structures [3, 4]; for using resonance as a sensing mechanism in micro and nano-devices [5]; for predicting the behavior of microdevices under temperature or stress variations [6,7]; for estimating the tension, residual stress and other physical parameters from the vibration response $[8,9]$.

Mechanical vibrations of the workpiece are analyzed using its modal shapes fundamentally and its frequency of oscillations that are uniformly distributed over it. Dynamic response of any structure design is addressed on its elements and an appropriate lightness of the structural requirements.

The relevance of this scientific issue is predetermined by a method to study the main parameters such as forces, displacements and stress in the supports of the structures or mechanical elements of machines. Also, to find the dynamic response under loads. The study results could be used to rationalize the design of modern structures.

\section{Literature review and problem statement}

The main problem with a clamping edge structure is that most of the dynamic analysis where it involves the oscillation frequencies of the structure only considers the elements where the forces act directly and do not analyze the clamped edge parts of the structure. Resonance of the structure, such as a cantilevered beam, is sometimes responsible for high values of response, even for small dynamic loads. Formulas have estimated these response frequencies instead of iteration or numerical processes, which has been the object of multiple works. 
In [8], the empirical equations are proposed to estimate the cable tension depending on the fundamental frequency of the cable, based on the solutions using the energy method and adjusting the exact solutions of the cable vibration equations where they take place in counts respectively the buckling and the bending stiffness. In this implementation, the cable clamped edge or clamping stress are not taken into account. After all, this is not required because the most in this analysis of the cables are the cable tensions themselves.

Carpinteri and Manuello [10] experimentally studied the evolution of the fundamental frequency in thin steel beams subjected to axial displacements at the ends. Three elements with different initial curvatures were tested under constraint conditions. The results obtained were that the fundamental frequency decreases with axial load. In this study, no analysis is performed on the clamping of the beams due to methodological circumstances.

This analysis is distinguished primarily by the beam assembly and testing procedure, primarily for the first beam constraint condition.

Zui and Namita [11] propose practical formulas for the vibration method taking into account the effects of bending stiffness and buckling of a cable. The practical formulas that the authors propose in this document are applicable to various cables, regardless of length and tension, to the extent that the first or second-order mode vibration can be measured. However, they do not address the analysis of cable clamp stresses due to low order modal shapes. The only formula is presented using natural frequencies of higher-order modes obtained from stationary micro-vibrations for very long cables.

In [12], closed-form solutions are presented for fundamental natural frequencies of non-homogeneous vibrating beams under axially distributed loads. The shape of the mode is postulated to coincide with the static deflection of the associated homogeneous beam without distributed axial load. In those solutions, the authors do not present embedment stress analysis as a relationship with modal stress distribution. They only present a linear relationship between the frequency and a load for all the boundary conditions.

\section{The aim and objectives of the study}

The aim of the study is to determine in an approximate way the clamped edge stresses that are generated in the clamping system due to the force applied to the workpiece to be machined through the analytical development proposed to calculate the maximum modal stress and then validate it with a numerical analysis using the main geometric and mechanical parameters of the workpiece.

To achieve the aim, the following objectives have been set:

- using the modal analysis to determine the natural frequencies and modal stresses that are distributed over the entire workpiece to be machined;

- to determine the clamped edge stress of the workpiece by the vibration amplitude of the generalized coordinate, using the differential equation of a single degree of freedom;

- numerically analyze the maximum clamped edge stress generated in the clamping system.

- to determine the displacement and the maximum clamping edge stress generated in the clamping system;

- to validate the case study using a finite element computer code.

\section{Materials and methods}

The maximum clamped edge stress is analyzed by the dynamic response using the modal analysis theory in a slender beam to determine the natural frequency of the system and its vibration forms. Subsequently, the numerical analysis is carried out with Ansys software using two materials for tools. The first, the clamping system, which is made of $\mathrm{H}-13$ steel with a yield stress of 1,380 MPa and the second a specimen of AISI 8620 steel, where the load is applied in the machining and considering the specimen in cantilever condition and using the constitutive relations and compatibility equations. The clamping system consists of two clamps that hold the material to work with a grip area of $3 \times 40$ millimeters in each of the clamps. Specimen data in a cantilever condition in analytical and numerical analysis are shown in Table 1.

Table 1

Data for the specimen to be machined

\begin{tabular}{|c|l|c|}
\hline \multicolumn{2}{|c|}{ Parameters } \\
\hline$L$ & Length $(\mathrm{m})$ & 0.2286 \\
\hline$b$ & Width $(\mathrm{m})$ & 0.0254 \\
\hline$h$ & Thickness $(\mathrm{m})$ & 0.0254 \\
\hline$E$ & Young Modulus $(\mathrm{GPa})$ & 205 \\
\hline$\rho$ & Mass Density $\left(\mathrm{Nsec}^{2} / \mathrm{m}^{4}\right)$ & 7,800 \\
\hline
\end{tabular}

4. 1. Modal analysis to determine the natural frequencies, modal shape and displacement of the workpiece

The dynamic response of the AISI 8620 steel specimen is analyzed to know stresses in the clamped edge. Fig. 1 shows the machining.

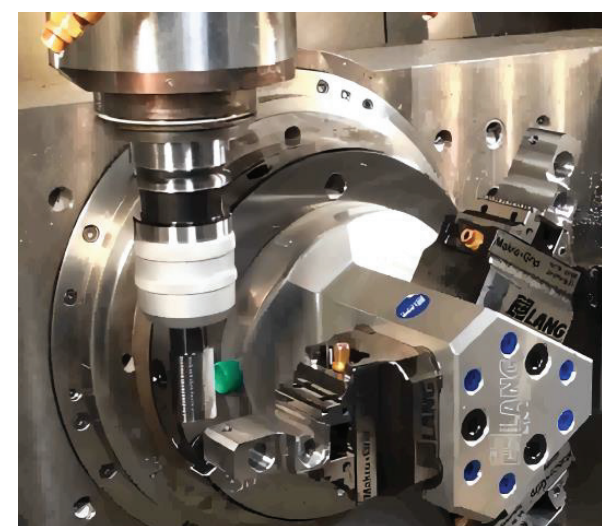

Fig. 1. Machining of the AISI 8620 steel specimen

The excitation force of amplitude $F_{0}=150 \mathrm{KN}$ and a variable frequency $\omega$ at a speed of 8,000 rpm. The primary differential equation (1) for a uniform beam where the deflections are a function of both time and location is [1]:

$$
E I \frac{\partial^{4} w(x, t)}{\partial x^{4}}=-\rho A \frac{\partial^{2} w(x, t)}{\partial u^{2}} .
$$

A result of a state of equilibrium between the elastic and the inertia forces in the area of the cross-section $A$, and uniform beam mass density $\rho$, so $\rho A$ is the mass per unit length of the specimen, assuming uniformity. It can be shown that 
the deflection $w(x, t)$ can be separated into two parts, one dependent on location only and the other on time:

$$
w(x, t)=\varphi(x) * \eta(t)
$$

Then two differential equations are obtained by modal function $\varphi(x)$ and generalized coordinate $\eta(t)$ :

$$
\frac{\partial^{4} \varphi(x)}{\partial x^{4}}-k_{n} \varphi(x)=0 \text {. }
$$

$K_{n}$ is associated with the resonance frequency $\omega_{n}$ :

$$
\begin{aligned}
& k_{n}^{4}=\frac{\omega_{n}^{2 *} \rho^{*} A}{E I} . \\
& \varphi_{n}\left(\frac{x}{L}\right)=\frac{1}{2}\left\{\begin{array}{l}
{\left[\cos \left(k_{n} * \frac{x}{L}\right)-\cosh \left(k_{n} * \frac{x}{L}\right)\right]+} \\
+\left[\frac{-\cos \left(k_{n} L\right)-\cosh \left(k_{n} L\right)}{\sin \left(k_{n} L\right)+\sinh \left(k_{n} L\right)}\right] \times \\
\times\left[\sin \left(k_{n} L * \frac{x}{L}\right)-\sinh \left(k_{n} L * \frac{x}{L}\right)\right]
\end{array}\right\} .
\end{aligned}
$$

The analysis for the first three natural frequencies of vibration calculated for the sample to be machined is shown in Table 2.

Table 2

Frequencies of analytical solutions

\begin{tabular}{|c|c|}
\hline \multicolumn{2}{|c|}{ Frequency $(\mathrm{Hz})$} \\
\hline Modes & Analytical \\
\hline 1 & 405.756 \\
\hline 2 & 2498.597 \\
\hline 3 & 5608.345 \\
\hline
\end{tabular}

In Fig. 2, the displacement of the first three mode shapes of the specimen of AISI 8620 is shown. It can be seen that the maximum absolute value of all the modes is $0.01 \mathrm{~m}$. This means that it is the displacement that the workpiece has due to the modal shape of vibration without having a previous load. The second mode has one additional nodal point, a location where the modal deflection is zero and the third mode has no deflection.

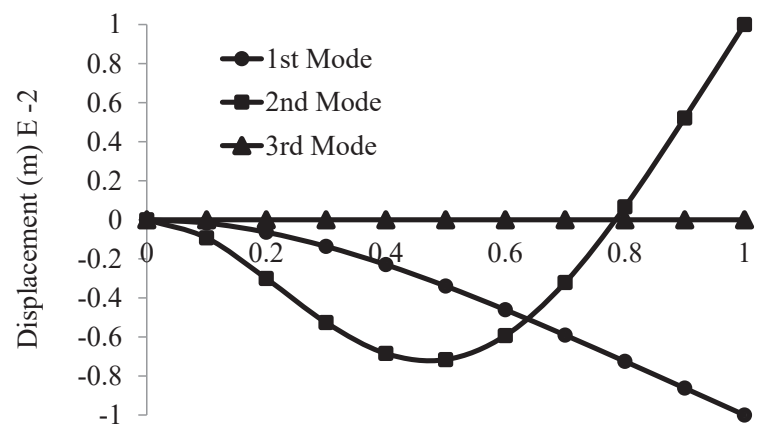

Fig. 2. Graph of the first three displacements of the AISI 8620 steel specimen

In the third modal form, the displacement is zero because the displacement is relatively minimal in such a way that it tends to zero. Fig. 2 shows that there is virtually no movement or displacement in the third modal shape of the specimen to be machined. The effect of the dynamic load factor (DLF) or the amplification factor is entered into the system when the deflections are calculated and serves to determine the final vibration amplitudes of the workpiece.

4.2. Development of an analytical model to determine the stress modes and clamped edge stress using the differential equation of a single degree of freedom

Stresses in an elastic structure are uniquely defined by the deflection, which means that deflections are obtained, either statically or dynamically. For a specific type of structure:

$$
\sigma_{i}=k L_{i}[w(x, y, t)] .
$$

$K$ is a constant depending on the material properties and geometry of the specimen:

$$
w \mathrm{r}(x, \mathrm{t})=\sum_{j=1}^{\infty} \varphi_{j}(x) * \eta_{j}(t) .
$$

From the previous equations, it is possible to write and propose a mathematical formulation that represents a linear function of the modal shapes and the generalized coordinates:

$$
\psi_{j}^{i}=K L_{i}\left[\varphi_{j}(x)\right] .
$$

The last equation (8) is for calculating the mode stresses $\psi_{j}^{i}$; there are also features of the structure. Thus, equation (8) is used to determine mode stresses in the clamping of the steel specimen. A modal superposition represents the lateral deflection of the specimen, so that a separation of variables is executed. The state of mode superposition is in effect that the total lateral displacement of the specimen is a weighted sum of the modal shapes. Thus, the displacement in any location and at any time is calculated:

$$
w(x, t)=\sum_{i=1}^{\infty} \varphi_{i}(x) * \eta_{i}(t)
$$

Differential equations of motion of a continuous system are performed, using Lagrange equations. For a system where the damping forces are derived from dissipation, the function:

$$
\frac{d}{d t}\left(\frac{d T}{d \eta_{r}}\right)-\frac{d T}{d \eta_{r}}+\frac{d U}{d \eta_{r}}+\frac{d D}{d \eta_{r}}=N_{r}
$$

where $T$ is the kinetic energy of the system, $U$ is the potential energy, $D$ is the dissipation function, and $N$ is the work done by the external force in the $\eta$ generalized coordinate. The development of the equation of motion is performed through a uniform specimen, for this reason, $m$ is the mass per unit measure, $E I$ is the flexural stiffness, $c$ is the damping per unit measure, and $\omega(x, t)$ is the displacement normal to the specimen along the $x$-axis. From equation (9):

$$
w^{\prime \prime}(x, t)=\sum_{i=1}^{\infty} \varphi_{i}^{\prime \prime}(x) * \eta(t) .
$$

Also, from equation (10):

$$
\frac{d T}{d \eta_{r}^{\prime}}=\int m w^{\prime} \frac{d w^{\prime}}{d \eta_{r}^{r}}=\int m \varphi_{r}(x)\left[\sum_{i=1}^{\infty} \varphi_{i}(x)^{*} \eta_{i}^{\prime}(t)\right] \mathrm{d} x .
$$


Being normal modes $\varphi_{j}$ and $\varphi_{i}$ are orthogonal, where $M_{r}$ is the $r$ th generalized mass, thus:

$$
\frac{d T}{d \eta_{r}^{\prime}}=\sum_{i=1}^{N} \eta_{i}^{\prime} \int m \varphi_{r}(x) \varphi_{i}(x) \mathrm{d} x=M_{r}
$$

To find the expression of the work done by the external force, some discrete force $f_{n}(t)$ must be applied to the specimen.

The virtual work done by the force in a virtual displacement, $\delta W(x, t)$ is:

$$
d w=\int p(t) \sum_{i=1}^{\infty} \varphi_{i}(x) d \eta_{i} d x=\sum_{i=1}^{\infty} d \eta_{i} \int p(t) \varphi_{i}(x) d x
$$

Substituting equation (13) into equation (10) yields a set of $N$ uncoupled differential equations for the generalized coordinate:

$$
M_{r} \eta_{r}^{\prime \prime}+2 \zeta_{r} \omega_{r} M_{r} \eta_{r}^{\mathrm{r}}+\omega_{r}^{2} M_{r} \eta_{r}=N_{r}
$$

From the complex equation of the transfer function, this means that there is a phase difference between excitation force and displacement:

$$
H(\Omega)=\frac{1}{\left(k-\Omega^{2} m\right)+i(\Omega c)} .
$$

This is the static deflection of a single degree of freedom under a static force. Therefore, the dynamic load factor is defined as:

$$
D L F=\frac{\left|x_{0}\right|}{x_{\text {static }}}=\frac{1}{\left[\left(1-\left(\frac{\Omega}{\omega_{0}}\right)^{2}+4\left(\frac{\Omega}{\omega_{0}}\right)^{2} \xi^{2}\right)\right]} .
$$

It is assumed that the damping ratio is $\zeta=2 \%$ [2]. The generalized mass of the first three resonance modes is calculated. The amplitude of the generalized coordinate is calculated using the differential equation of a single degree of freedom and the dynamic load factor at its maximum value.

Like the displacements in the third modal shape, the modal stresses for the same shape are tiny and ideally tend to zero shown in Table 3 and Fig. 3.

This means that the maximum modal forces are only between the first and second modal shape of the specimen to be machined. To obtain the stress modes, one naturally tends to use equation (8), i. e., to apply the operator $L$ on the modal shape.

Table 3

Analytical solutions

\begin{tabular}{|c|c|}
\hline \multicolumn{2}{|c|}{ Maximum stress modes (MPa) } \\
\hline Modes & Analytical \\
\hline 1 & 1777.245 \\
\hline 2 & 39457.857 \\
\hline 3 & 0 \\
\hline
\end{tabular}

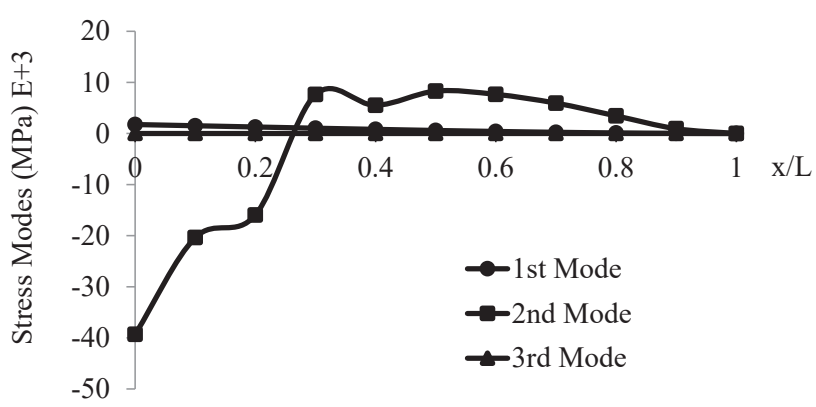

Fig. 3. Graph of the first three modal stresses of the AISI 8620 steel specimen

4. 3. Numerical analysis to determine the displacement and the maximum clamping edge stress generated in the clamping system

Finite Element Method (FEM) model was built according to the dimensions of the clamping system and the machined material. The simulation process consists of determining the load imposed on the clamps of the $\mathrm{H}-13$ steel system due to the point force at the end of the AISI 8620 steel specimen that is exerted when machining. The model of Fig. 4 is considered with isotropic, homogeneous, continuous, and linear properties.

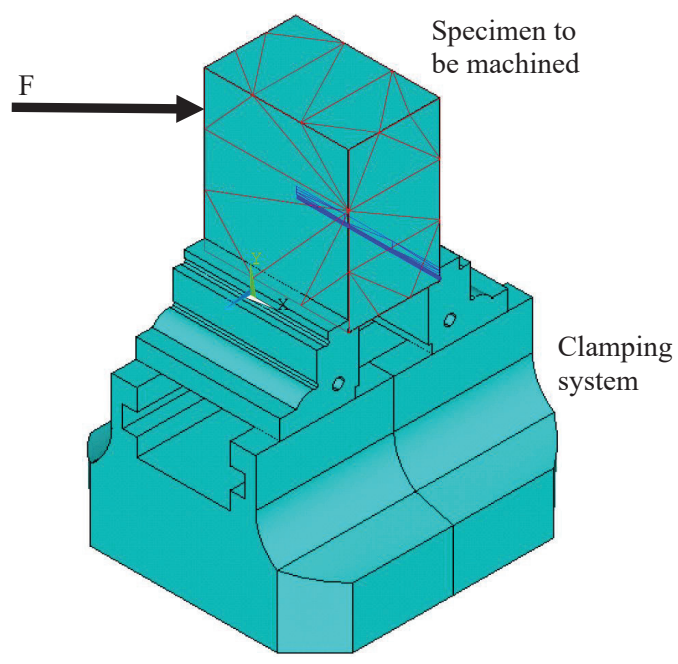

Fig. 4. H-13 steel material clamping system and AISI 8620 steel workpiece

Subsequently, the displacement and the maximum camped edge stress generated in the clamping system for the first three modal shapes are determined using modal analysis. Higher-order 3D elements are used. SOLID186 element is defined by 20 nodes with three degrees of freedom per node, translation in $X, Y$ and $Z$. Only one jaw of the specimen has been modelled, due to symmetry (with respect to $X-Z$ and $X-Y$ Cartesian planes) and symmetric displacement boundary condition has been applied to the corresponding planes and is shown in Fig. 5. The stress distribution in the jaw is obtained, a three-dimensional finite element model was simulated by ANSYS $^{\circledR}$ general finite element code. The meshed model of the specimen to be machined consisted of 19,785 nodes and the clamp consisted of 44,750 nodes.

The Poisson ratio NUXY $=0.3$, elastic modulus $E=$ $=2.1 \cdot 10^{5} \mathrm{MPa}$, material density DENS $=7,800 \mathrm{Nsec}^{2} / \mathrm{m}^{4}$. The most critical holding areas were with a complex mesh. 


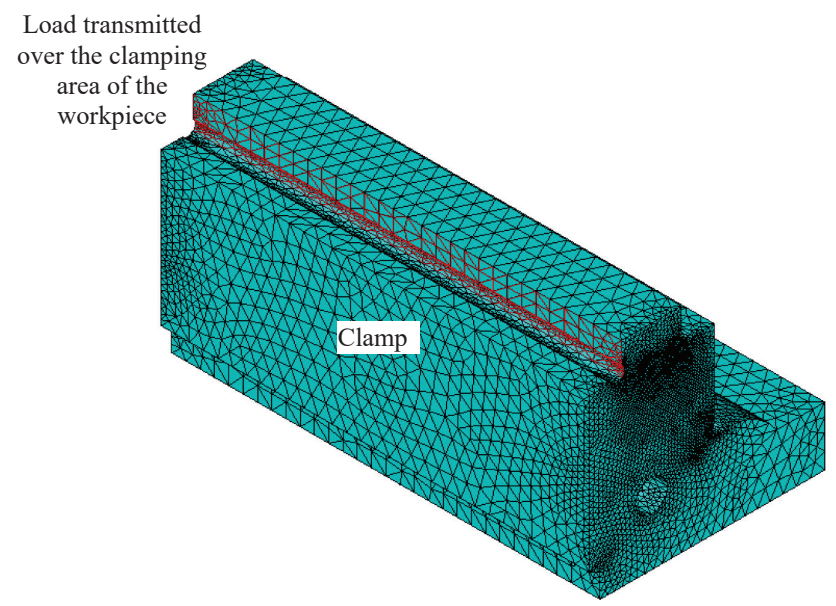

Fig. 5. Clamp with free meshing

The analysis was developed into natural frequencies, displacement and stress in the clamping system and has approximated visualization of the effects of applying a harmonic excitation on the specimen. The load imposed on the specimen to be machined is transmitted in the jaw system, as shown in Fig. 5. Jaw displacement is shown when pressure is applied to the workpiece clamping area, as in Fig. 6 . The boundary constraints assignment (offset) is considered subject at the base of the clamping system.

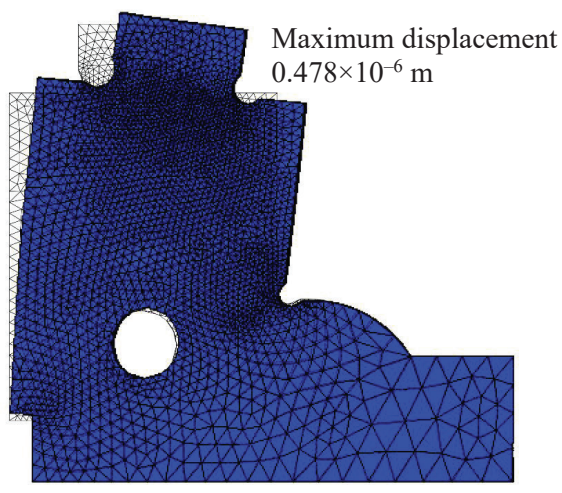

Fig. 6. Clamp displacement

The stresses are generated in areas where the model geometry is critical; that is, the slack is quite pronounced. Therefore, those stresses are shown in Fig. 7.

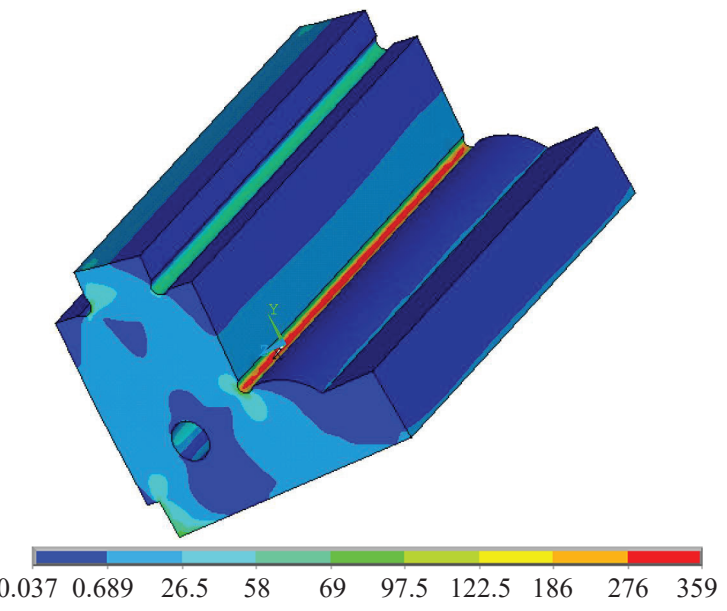

Fig. 7. Maximum stress in the critical zone
The maximum values of the generalized coordinate or amplitude for the first, second and third modes are shown in Table 4. Note that the first response is in a wide frequency between 350 and $720 \mathrm{~Hz}$, a range that includes the first frequency computed.

Table 4

Numerical solutions of the ending amplitude

\begin{tabular}{|c|c|}
\hline Mode & Numerical \\
\hline 1 & 0.002087 \\
\hline 2 & 0.000053 \\
\hline 3 & 0 \\
\hline
\end{tabular}

The expression for the total generalized coordinate should include the phase angle between the response and excitation, as shown in Fig. 8, 9.

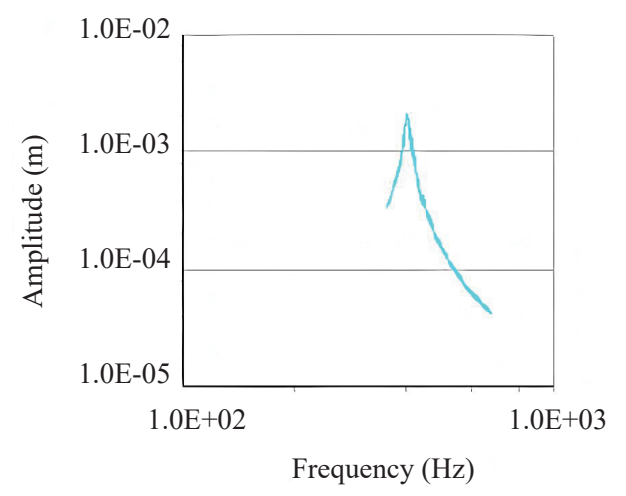

Fig. 8. Amplitude of the end of the specimen

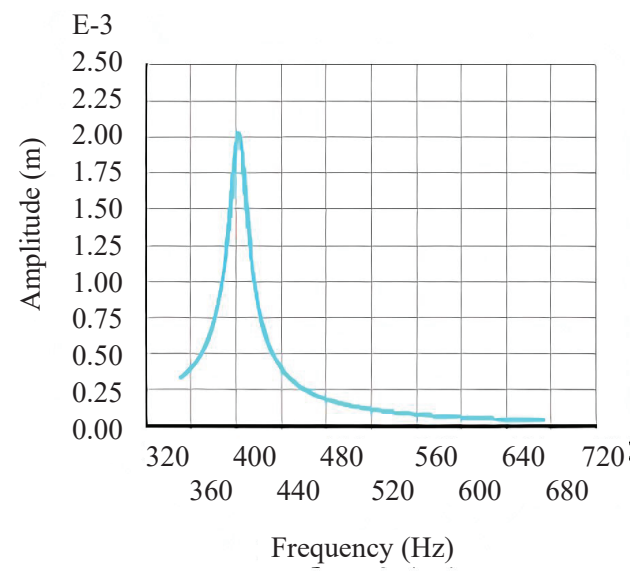

Fig. 9. Generalized coordinate for the first mode at the specimen end

The study also has shown that the amplitude or displacement of the workpiece that is observed in Fig. 9 and Table 4 where its value is 0.002080 meters, being limited to one centimeter, and finally the estimation of stress due to the imposed load on the clamp must be it is $358 \mathrm{MPa}$, as shown in Fig. 10, which is less than the yield strength of both materials.

The numerical analysis of the stresses at the clamped edge of the first three modal shapes of the clamping system is shown in Table 5 and Fig. 10. 


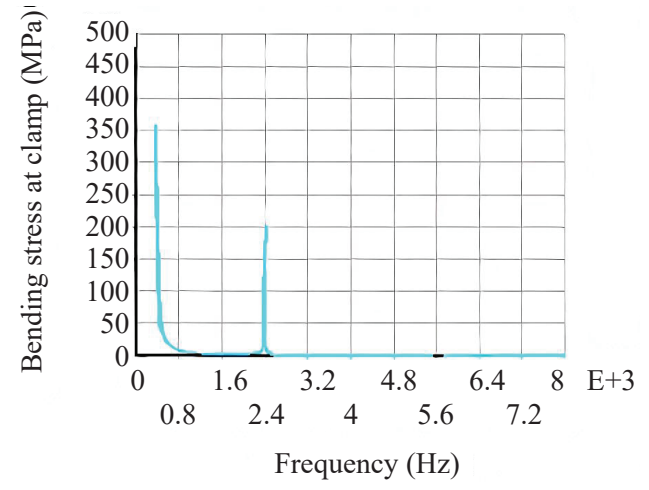

Fig. 10. Clamped edge stress in the clamping system calculated from the numerical solution

Table 5

Numerical solutions of clamped edge stress

\begin{tabular}{|c|c|}
\hline Mode & Numerical \\
\hline 1 & 359.0 \\
\hline 2 & 202.0 \\
\hline 3 & 0 \\
\hline
\end{tabular}

\section{Discussion for the clamped edge stress in a structural element under cantilever condition}

The analysis proposed to estimate the clamped edge stress in the cantilever structures, which could be a new way of knowing the dynamic response of a structure under this support condition.

This is evidenced by the analytical model proposed under the theory of modal analysis. The results obtained can be explained by the possibility of applying the Lagrange equations to the calculation of mechanical structures, taking into account the use of energy in a mechanical system or structure.

The presence of modal stresses in the structure leads to an analytical formulation to be able to estimate the clamped edge stress of the clamping system. Due to equation (8), it is possible to calculate the stress mode, which is a parameter that characterizes the structure and shows that the modal stress is a stress distribution throughout the structure when subjected to a deflection equal to the modal shape.

When analyzing the displacement compartment, it is observed that the first displacement mode dominates the response and that the forces due to the first mode are practically those that influence the final result of the embedment forces, as shown in Fig. 2, 3.

Fundamentally, the method to calculate clamped edge stress algorithmically in six steps was implemented in this study.

To implement this method in actual practice for structures subjected to a cantilevered support condition, it is needed to address the following main issues:

1) calculate the resonance frequencies and mode shape. These can be done analytically for simple structural elements or numerically using a fine element computer program;

2) calculate generalized masses using equation (13);
3) knowing the external loading, calculate the generalized forces of the system using equation (14).

4) assume modal damping coefficients $\zeta$. These are known from the nature of the mode.

5 ) solve $N$ uncoupled differential equations for the generalized coordinate.

6) calculate the stress modes behavior using equation (8). Thus, this equation is used to determine modal stresses in the clamping of the steel specimen.

Difficulties in conducting studies on mathematical analysis are mainly related to the following two issues:

1) the application of the operator $L$ is not always known in closed form, although it is possible to express it for simple cases.

2) the modes shapes of the structure are not always available in a closed-form expression. In many cases, the modes are approximated by assumed expressions that satisfy the boundary conditions and give a good approximation for the resonance frequencies.

However, these difficulties can be significantly reduced by using the numerical analysis, which simulates the movement and clamps edge stress of the clamping system based on a combination of results to solve the Lagrange's differential equation and the equations of motion of a point mass, taking into account the vibration forms, frequencies and modal forms of the system.

\section{Conclusions}

1. The development of equations of motion is performed for a beam as a continuous system using Lagrange equations. Because the modal superposition represents the lateral deflection of the beam, so, any deflection is a superposition of a modal function that depends on the position and the generalized coordinate that depends on the time, where the weighting functions are the general coordinates.

2 . The proposed study showed that the modal analysis determines the natural frequency and modal stress. Modal stress is a characteristic of the structure and not of the load condition, in such a way that it is distributed throughout the structure when the latter is subjected to a deflection statically equal to the modal shape, with the generalized coordinate normalized to one centimeter, as shown in Fig. 2.

3. The actual stresses at the clamped edge of the structure can be obtained by multiplying values of the stress modes at that edge with the deflection of the structure tip. According to the dynamic analysis of the clamping load made on an $\mathrm{H}-13$ steel material, it supports the harmonic force imposed by the machining. The maximum stresses are near the clamped edge, where usually no accelerations exist. It is interesting to note that the structural locations that experience the largest amplitudes are not necessarily those in which maximum stresses exist, as shown in Fig. 3.

4. The validation of the analytical method is carried out through the modal analysis of the LANCZOS method, which is the most efficient way to solve the eigenvalue. Moreover, the BLOCKLANCZOS method is selected for the characteristics of high precision and computing speed. Natural frequencies, stresses and displacements are determined, based on the Finite Element Method, such as ANSYS ${ }^{\circledR}$ software. In the first three natural frequencies of the workpiece, there 
is an approximate variation of $4.7 \%$ between both analytical and numerical methods. Fig. 3 shows the modal stress with a maximum value of $1,700 \mathrm{MPa}$. It was found that the maximum modal stress is 1,700 MPa and highlighted that these stresses are considered only in the first two modal shapes, and for the third, the modal stresses are minimal and practically tend to zero.

\section{Acknowledgments}

The authors gratefully acknowledge the financial support from the Mexican government by the Consejo Nacional de Ciencia y Tecnología. The authors acknowledge partial support of projects 20201964, 20200930 and 20200305, as well as an EDI grant, all by SIP/IPN.

\section{References}

1. Bokaian, A. (1988). Natural frequencies of beams under compressive axial loads. Journal of Sound and Vibration, 126 (1), 49-65. doi: https://doi.org/10.1016/0022-460x(88)90397-5

2. Hou, Z., Xiao, D., Wu, X., Dong, P., Chen, Z., Niu, Z., Zhang, X. (2010). Effect of Axial Force on the Performance of Micromachined Vibratory Rate Gyroscopes. Sensors, 11 (1), 296-309. doi: https://doi.org/10.3390/s110100296

3. Gillich, G.-R., Furdui, H., Abdel Wahab, M., Korka, Z.-I. (2019). A robust damage detection method based on multi-modal analysis in variable temperature conditions. Mechanical Systems and Signal Processing, 115, 361-379. doi: https://doi.org/10.1016/j.ymssp.2018.05.037

4. Du, X., Wang, L., Li, A., Wang, L., Sun, D. (2017). High Accuracy Resonant Pressure Sensor With Balanced-Mass DETF Resonator and Twinborn Diaphragms. Journal of Microelectromechanical Systems, 26 (1), 235-245. doi: https://doi.org/10.1109/jmems.2016.2632108

5. Hu, Y., Xue, H., Hu, H. (2007). A piezoelectric power harvester with adjustable frequency through axial preloads. Smart Materials and Structures, 16 (5), 1961-1966. doi: https://doi.org/10.1088/0964-1726/16/5/054

6. Blevins, R. D., Plunkett, R. (1980). Formulas for Natural Frequency and Mode Shape. Journal of Applied Mechanics, 47 (2), $461-462$. doi: https://doi.org/10.1115/1.3153712

7. Zhou, P., Hu, Y., Zhang, X. (2005). Based on ANSYS modal analysis for gas compressor disc. Machinery Design \& Manufacture, 6, 61-62.

8. Ren, W.-X., Chen, G., Hu, W.-H. (2005). Empirical formulas to estimate cable tension by cable fundamental frequency. Structural Engineering and Mechanics, 20 (3), 363-380. doi: https://doi.org/10.12989/sem.2005.20.3.363

9. Yesilce, Y., Demirdag, O. (2008). Effect of axial force on free vibration of Timoshenko multi-span beam carrying multiple springmass systems. International Journal of Mechanical Sciences, 50 (6), 995-1003. doi: https://doi.org/10.1016/j.ijmecsci.2008.03.001

10. Carpinteri, A., Malvano, R., Manuello, A., Piana, G. (2014). Fundamental frequency evolution in slender beams subjected to imposed axial displacements. Journal of Sound and Vibration, 333 (11), 2390-2403. doi: https://doi.org/10.1016/j.jsv.2014.01.018

11. Zui, H., Shinke, T., Namita, Y. (1996). Practical Formulas for Estimation of Cable Tension by Vibration Method. Journal of Structural Engineering, 122 (6), 651-656. doi: https://doi.org/10.1061/(asce)0733-9445(1996)122:6(651)

12. Gellert, M., Gluck, J. (1972). The influence of axial load on eigen-frequencies of a vibrating lateral restraint cantilever. International Journal of Mechanical Sciences, 14 (11), 723-728. doi: https://doi.org/10.1016/0020-7403(72)90010-0 